\title{
Parabolic Verma Modules and Invariant Differential Operators ${ }^{1}$
}

\author{
V.K. Dobrev \\ Institute of Nuclear Research and Nuclear Energy, \\ Bulgarian Academy of Sciences, \\ 72 Tsarigradsko Chaussee, 1784 Sofia, Bulgaria
}

\begin{abstract}
In the present paper we continue the project of systematic classification and construction of invariant differential operators for non-compact semisimple Lie groups. This time we make the stress on one of the main building blocks, namely the Verma modules and the corresponding parabolic subalgebras. In particular, we start the study of the relation between the parabolic subalgebras of real semisimple Lie algebras and of their complexification. Two cases are given in more detail: the conformal algebra of 4D Minkowski space-time and the minimal parabolics of classical real semisimple Lie algebras.
\end{abstract}

\section{Introduction}

Verma modules play crucial role in the classification and construction of invariant differential operators for non-compact semisimple Lie groups. In our approach we make important use of the relations between the parabolic subalgebras of a real semisimple Lie algebra $\mathcal{G}_{0}$ and those of its complexification $\mathcal{G}$. It the present paper we make this relation more transparent briefly in general and in some detail in examples.

\section{Preliminaries}

Let $G$ be a semisimple non-compact Lie group, and $K$ a maximal compact subgroup of $G$. Then we have the Iwasawa decomposition $G=K A_{0} N_{0}$, where $A_{0}$ is Abelian simply connected vector subgroup of $G, N_{0}$ is a nilpotent simply connected subgroup of $G$ preserved by the action of $A_{0}$. Further, let $M_{0}$ be the centralizer of $A_{0}$ in $K$. Then the subgroup $P_{0}=M_{0} A_{0} N_{0}$ is a minimal parabolic subgroup of $G$. A parabolic subgroup $P=M A N$ is any subgroup of $G$ which contains a minimal parabolic subgroup. In the general case $A \subset A_{0}$ is abelian, $N \subset N_{0}$ is a nilpotent simply connected subgroup of $G$ preserved by the action of $A, M \supset M_{0}$ is a maximal centralizer of $A$ in $G$.

Further, let $\mathcal{G}_{0}, \mathcal{K}, \mathcal{P}, \mathcal{M}, \mathcal{A}, \mathcal{N}$ denote the Lie algebras of $G, K, P, M, A, N$, resp.

We note also another extremal case : maximal parabolic subgroup when rank $A=1$, maximal parabolic subalgebra when $\operatorname{dim} \mathcal{A}=1$.

Let $\nu$ be a (non-unitary) character of $A, \nu \in \mathcal{A}^{*}$. Let $\mu$ fix a finite-dimensional (non-unitary) representation $D^{\mu}$ of $M$ on the space $V_{\mu}$. In the case when $M$ is cuspidal then we may use also the discrete series representation of $M$ with the same Casimirs as $D^{\mu}$.

\footnotetext{
${ }^{1}$ Plenary talk at Bogolyubov Conference, Moscow-Dubna, 11.9.2019.
} 
We call the induced representation $\chi=\operatorname{Ind}_{P}^{G}(\mu \otimes \nu \otimes 1)$ an elementary representation of $G$ [1]. (These are called generalized principal series representations (or limits thereof) in [2].) Their spaces of functions are:

$$
\mathcal{C}_{\chi}=\left\{\mathcal{F} \in C^{\infty}\left(G, V_{\mu}\right) \mid \mathcal{F}(\text { gman })=e^{-\nu(H)} \cdot D^{\mu}\left(m^{-1}\right) \mathcal{F}(g)\right\}
$$

where $a=\exp (H) \in A, H \in \mathcal{A}, m \in M, n \in N$. The representation action is the left regular action:

$$
\left(\mathcal{T}^{\chi}(g) \mathcal{F}\right)\left(g^{\prime}\right)=\mathcal{F}\left(g^{-1} g^{\prime}\right), \quad g, g^{\prime} \in G .
$$

\section{Verma modules}

An important ingredient in our considerations are the highest/lowest weight representations of $\mathcal{G}$, where $\mathcal{G}$ is the complexification of $\mathcal{G}_{0}$. These can be realized as (factor-modules of) Verma modules $V^{\Lambda}$ over $\mathcal{G}$, where $\Lambda \in(\mathcal{H})^{*}, \mathcal{H}$ is a Cartan subalgebra of $\mathcal{G}$, the weight $\Lambda=\Lambda(\chi)$ is determined uniquely from $\chi[3]$.

We recall that a Verma module $[4,5]$ is a $\mathcal{G}$-module induced from a character of a Borel subalgebra $\mathcal{B}=\mathcal{H} \oplus \mathcal{N}$. (We use the standard triangular decomposition $\mathcal{G}=\mathcal{N} \oplus \mathcal{H} \oplus \mathcal{N}^{-}$.) Let $1_{\Lambda}$ denote the $\mathcal{B}$-module $1_{\Lambda}=\mathbb{C} v_{0}$ such that $\mathcal{N} v_{0}=0, H v_{0}=\Lambda(H) v_{0}, H \in \mathcal{H},\left(\operatorname{dim} 1_{\Lambda}=1\right)$. Let $V^{\Lambda}$ be the corresponding Verma module, then

$$
V^{\Lambda}=U(\mathcal{G}) \otimes_{U(\mathcal{B})} 1_{\Lambda}
$$

Using

$$
U(\mathcal{G})=U\left(\mathcal{N}^{-}\right) \otimes U(\mathcal{B})
$$

one has

$$
V^{\Lambda}=U\left(\mathcal{N}^{-}\right) \otimes 1_{\Lambda} .
$$

Obviously $V^{\Lambda}$ is a highest weight module with highest weight $\Lambda$, and highest weight vector $v_{0}$, $V^{\Lambda} \cong U\left(\mathcal{N}^{-}\right)$as vector spaces.

Actually, since our ERs are induced from finite-dimensional representations of $\mathcal{M}$ (or their limits) the Verma modules are always reducible. Thus, it is more convenient to use generalised Verma modules $\tilde{V}^{\Lambda}$ such that the role of the highest/lowest weight vector $v_{0}$ is taken by the (finite-dimensional) space $V_{\mu} v_{0}$.

It is important to note that the generalized Verma modules defined just above and related to ERs are special cases of parabolic Verma modules (PVM) which are introduced in purely algebraic context. More precisely, their construction is as follows. (Below we define PVM using [6], except that there these modules are called generalized Verma modules (which in our approach is used differently, see above and [7]).

Let $\Delta$ be the root system of $(\mathcal{G}, \mathcal{H}), \Delta_{+}, \Delta_{-}$denote the positive, negative, roots. Let $\alpha_{i} \in$ $\mathcal{H}^{*}$ be the simple roots where $i=1, \ldots, \ell=\operatorname{dim} \mathcal{H}$. Let $e_{i}$ (resp. $f_{i}$ ) be a non-zero element of the root space $\mathcal{G}_{\alpha_{i}}$ (resp. $\mathcal{G}_{-\alpha_{i}}$ ) for all $i=1, \ldots, \ell$ normalized so that $\left[e_{i}, f_{i}\right]=h_{i}$, and $\alpha_{i}\left(h_{i}\right)=2$. Let $S$ be a subset of the set $J=\{1, \ldots, \ell\}$. Let $\mathcal{G}_{S}$ be the subalgebra of $\mathcal{G}$ generated by $\left\{h_{i}, e_{i}, f_{i}\right\}_{i \in S} ; \mathcal{H}_{S}$ the span of $\left\{h_{i}\right\}_{i \in S} ; \Delta^{S}=\Delta \cap \amalg_{i \in S} \mathbb{Z} \alpha_{i} ; \Delta_{ \pm}^{S}=\Delta_{ \pm} \cap \Delta^{S}$; $\Delta(S)_{ \pm}=\Delta_{ \pm}-\Delta_{ \pm}^{S}$. 
Further, we define the following subalgebras of $\mathcal{G}: \mathcal{N}=\amalg_{\beta \in \Delta_{+}} \mathcal{G}^{\beta} ; \mathcal{N}^{-}=\amalg_{\beta \in \Delta_{-}} \mathcal{G}^{\beta}$ (the latter two were already used above); $\mathcal{N}_{S}=\amalg_{\beta \in \Delta_{+}^{S}} \mathcal{G}^{\beta} ; \mathcal{N}_{S}^{-}=\amalg_{\beta \in \Delta_{-}^{S}} \mathcal{G}^{\beta} ; \mathcal{U}_{S}=\amalg_{\beta \in \Delta_{+}(S)} \mathcal{G}^{\beta}$; $\mathcal{U}_{S}^{-}=\amalg_{\beta \in \Delta_{-}(S)} \mathcal{G}^{\beta} ; \mathcal{R}_{S}=\mathcal{G}_{S} \oplus \mathcal{H} ; \mathcal{P}_{S}=\mathcal{R}_{S} \oplus \mathcal{U}_{S}$.

Then we have: $\mathcal{G}=\mathcal{N} \oplus \mathcal{H} \oplus \mathcal{N}^{-}, \mathcal{G}_{S}=\mathcal{N}_{S} \oplus \mathcal{H}_{S} \oplus \mathcal{N}_{S}^{-} ; \mathcal{N}=\mathcal{N}_{S} \oplus \mathcal{U}_{S} ; \mathcal{N}^{-}=$ $\mathcal{N}_{S}^{-} \oplus \mathcal{U}_{S}^{-} ; \mathcal{R}_{S}=\mathcal{N}_{S} \oplus \mathcal{H} \oplus \mathcal{N}_{S}^{-} ; \mathcal{G}=\mathcal{U}_{S}^{-} \oplus \mathcal{P}_{S}$

Further, $\mathcal{G}_{S}$ is a split semisimple Lie algebra with splitting Cartan subalgebra $\mathcal{H}_{S} ; \mathcal{R}_{S}$ is a reductive Lie algebra with commutator subalgebra $\mathcal{G}_{S}$ and centre a subalgebra of $\mathcal{H}$.

As $S$ varies among the subsets of $J, \mathcal{P}_{S}$ varies among the parabolic subalgebras of $\mathcal{G}$ containing the Borel subalgebra $\mathcal{B}=\mathcal{H} \oplus \mathcal{N}$. The reductive part of $\mathcal{P}_{S}$ is $\mathcal{R}_{S}$ and the nilpotent part of $\mathcal{P}_{S}$ is $\mathcal{U}_{S}$. We note that if $S=\emptyset$, then $\mathcal{P}_{\emptyset}=\mathcal{B}\left(\right.$ since $\left.\mathcal{G}_{\emptyset}=0, \mathcal{R}_{\emptyset}=\mathcal{H}, \mathcal{U}_{\emptyset}=\mathcal{N}\right)$.

Now let $P$ indexes the set of (equivalent classes of) finite-dimensional irreducible $\mathcal{G}$-modules in the usual way - via the highest weight. Let $P_{S}=\left\{\lambda \in \mathcal{H}^{*} \mid \lambda\left(h_{i}\right) \in \mathbb{Z}_{+}\right.$for all $\left.i \in S\right\}$. Then it is clear that there is a natural bijection, $\lambda \mapsto M(\lambda)$, between $P_{S}$ and the set of (equivalent classes of) finite-dimensional irreducible $\mathcal{R}_{S}$-modules which are irreducible as $\mathcal{G}_{S}$-modules.

For any $\lambda \in P_{S}$, denote by $V_{S}^{M(\lambda)}$ the corresponding parabolic Verma module (PVM) : the $\mathcal{G}$-module $\operatorname{ind}(M(\lambda), \mathcal{G})$ induced by the $P_{S}$-module $M(\lambda)$, viewed as an $\mathcal{R}_{S}$-module in the natural way and as a trivial $\mathcal{U}_{S}$-module.

Note that for $S=\emptyset$ the parabolic Verma modules coincide with the usual Verma modules : $V_{\emptyset}^{M(\lambda)}=V^{\lambda}$.

Further we discuss the reducibility of Verma modules.

A classic result of [8] states that a Verma module $V^{\Lambda}$ is reducible iff

$$
\left(\Lambda+\rho, \beta^{\vee}\right)=m, \quad m \in \mathbb{N}, \beta \in \Delta^{+},
$$

where $\beta^{\vee} \equiv 2 \beta /(\beta, \beta), \rho$ is half the sum of the positive roots of $\mathcal{G}$.

The same criterion of reducibility is valid for generalized Verma modules, though it is trivially satisfied for the $\mathcal{M}$-compact roots, and is essential only for $\mathcal{M}$-non-compact roots. (We recall that $\mathcal{M}$-compact roots are those elements of $\Delta$ that belong to the root system of $\mathcal{M}^{\mathbb{C}}$, the latter being identified as a subset of $\Delta$.)

The same criterion of reducibility is valid for a parabolic Verma module $V_{S}^{M(\Lambda)}$ though it is trivially satisfied for $\beta \in \Delta_{+}^{S}$, and is essential only for $\beta \in \Delta_{+}(S)$.

When (6) holds then the Verma module with shifted weight $V^{\Lambda-m \beta}$ (or $\tilde{V}^{\Lambda-m \beta}$ for GVM and $\beta \mathcal{M}$-non-compact, or $V_{S}^{M(\Lambda-m \beta)}$ for PVM and $\left.\beta \in \Delta_{+}(S)\right)$ is embedded in the Verma module $V^{\Lambda}$ (or $\tilde{V}^{\Lambda}$, or $\left.V_{S}^{M(\Lambda)}\right)$.

The above embedding is realized by a singular vector $v_{s}$ determined by a polynomial $\mathcal{P}_{m, \beta}\left(\mathcal{N}^{-}\right)$in the universal enveloping algebra $\left(U\left(\mathcal{N}^{-}\right)\right) v_{0}$. More explicitly, [3], $v_{m, \beta}^{s}=$ $\mathcal{P}_{m, \beta} v_{0}$. Relatedly, then there exists [3] an intertwining differential operator

$$
\mathcal{D}_{m, \beta}: \mathcal{C}_{\chi(\Lambda)} \longrightarrow \mathcal{C}_{\chi(\Lambda-m \beta)}
$$

given explicitly by:

$$
\mathcal{D}_{m, \beta}=\mathcal{P}_{m, \beta}\left(\widehat{\mathcal{N}^{-}}\right)
$$

where $\widehat{\mathcal{N}^{-}}$denotes the right action on the functions $\mathcal{F}$.

In the next Section we shall consider the example of the conformal algebra. 


\section{Conformal algebra}

The conformal algebra in four-dimensional space-time is $\mathcal{G}_{0}=s u(2,2)(\cong s o(4,2))$. It has three nonconjugate parabolic subalgebras $(\mathcal{P}=\mathcal{M} \oplus \mathcal{A} \oplus \mathcal{N})$ :

$$
\begin{aligned}
\mathcal{P}_{0}= & \operatorname{so}(2) \oplus \mathcal{A}_{0} \oplus \mathcal{N}_{0}, \\
& \operatorname{dim} \mathcal{A}_{0}=2, \quad \operatorname{dim} \mathcal{N}_{0}=6, \\
\mathcal{P}_{1}= & \operatorname{so}(2) \oplus \operatorname{sl}(2, \mathbb{R}) \oplus \mathcal{A}_{1} \oplus \mathcal{N}_{1}, \\
& \operatorname{dim} \mathcal{A}_{1}=1, \quad \operatorname{dim} \mathcal{N}_{1}=5, \\
\mathcal{P}_{2}= & \operatorname{so}(3,1) \oplus \mathcal{A}_{2} \oplus \mathcal{N}_{2}, \\
& \operatorname{dim} \mathcal{A}_{2}=1, \quad \operatorname{dim} \mathcal{N}_{2}=4
\end{aligned}
$$

where $\mathcal{P}_{0}$ is the minimal parabolic, $\mathcal{P}_{1}$ is maximal cuspidal, $\mathcal{P}_{2}$ is maximal noncuspidal.

\subsection{Maximal non-cuspidal case}

We consider the following Bruhat decomposition [9] (consistent with the maximal non-cuspidal parabolic subalgebra $\mathcal{P}_{2}$ ):

$$
\mathcal{G}_{0}=\mathcal{G}_{0}^{+} \oplus \mathcal{M}_{2} \oplus \mathcal{A}_{2} \oplus \mathcal{G}_{0}^{-}
$$

where $\mathcal{M}_{2}$ is the six-dimensional Lorentz subalgebra $s o(3,1), \mathcal{A}_{2}$ is the dilatation subalgebra, $\mathcal{G}_{0}^{+}, \mathcal{G}_{0}^{-}$is the four-dimensional isomorphic translation subalgebra, resp., special conformal transformations subalgebra.

In this case the ERs of $s u(2,2)$ are parametrized by triples: $\chi=\left[j_{1}, j_{2} ; d\right]$, where $j_{1}, j_{2} \in$ $\frac{1}{2} \mathbb{Z}_{+}$parametrize the finite-dimensional representations of $\mathcal{M}_{2}$, while the number $d$ parametrizing the representations of $\mathcal{A}_{2}$ is called the conformal weight or energy.

The complexificaton of $\mathcal{G}_{0}$ is $\mathcal{G}=\operatorname{sl}(4)$. The root system of $\mathcal{G}$ is given by:

$$
\Delta^{+}=\left\{\alpha_{1}, \alpha_{2}, \alpha_{3}, \alpha_{12}=\alpha_{1}+\alpha_{2}, \alpha_{12}=\alpha_{1}+\alpha_{2}, \alpha_{23}=\alpha_{2}+\alpha_{3}, \alpha_{13}=\alpha_{1}+\alpha_{2}+\alpha_{3}\right\}
$$

where $\left\{\alpha_{1}, \alpha_{2}, \alpha_{3}\right\}$ are the simple roots.

Note that when relating the root systems of $\mathcal{G}$ to $\mathcal{G}_{0}$ relative to the Bruhat decomposition (10) the roots $\left\{\alpha_{1}, \alpha_{3}\right\}$ are $\mathcal{M}_{2}$-compact, the rest are $\mathcal{M}_{2}$-non-compact.

The reducibility conditions (6) of a Verma module $V^{\Lambda}$ over $\mathcal{G}$ are written explicitly as follows:

$$
\begin{aligned}
m_{1} & =\left(\Lambda+\rho, \alpha_{1}\right) \in \mathbb{N} \\
m_{2} & =\left(\Lambda+\rho, \alpha_{2}\right) \in \mathbb{N} \\
m_{3} & =\left(\Lambda+\rho, \alpha_{3}\right) \in \mathbb{N} \\
m_{12} & =\left(\Lambda+\rho, \alpha_{12}\right)=m_{1}+m_{2} \in \mathbb{N} \\
m_{23} & =\left(\Lambda+\rho, \alpha_{23}\right)=m_{2}+m_{3} \in \mathbb{N} \\
m_{13} & =\left(\Lambda+\rho, \alpha_{13}\right)=m_{1}+m_{2}+m_{3} \in \mathbb{N}
\end{aligned}
$$


We want to apply these conditions to the signatures $\chi$ of the ERs. In these terms we have [7]:

$$
\begin{aligned}
m_{1} & =2 j_{1}+1 \in \mathbb{N} \\
m_{2} & =2-d-j_{1}-j_{2} \in \mathbb{N} \\
m_{3} & =2 j_{2}+1 \in \mathbb{N} \\
m_{12} & =3-d+j_{1}-j_{2} \in \mathbb{N} \\
m_{23} & =3-d-j_{1}+j_{2} \in \mathbb{N} \\
m_{13} & =4-d+j_{1}+j_{2} \in \mathbb{N}
\end{aligned}
$$

Note that (12a,c) are fulfilled always since $2 j_{1}+1 \in \mathbb{N}, 2 j_{2}+1 \in \mathbb{N}$, as expected for the $\mathcal{M}_{2}$-compact roots. On the other hand the expressions in the other cases depend on $d$ and may be arbitrary.

Note that $m_{i}$ considered abstractly are called Dynkin labels, while together with $m_{i j}$ they are called Harish-Chandra parameters [10]:

$$
m_{\beta} \equiv(\Lambda+\rho, \beta)
$$

where $\beta$ is any positive root of $\mathcal{G}$. These parameters are redundant, since they are expressed in terms of the Dynkin labels, however, some statements are best formulated in their terms.

\subsection{Maximal cuspidal case}

We consider again the conformal algebra $\mathcal{G}_{0}=s u(2,2)(\cong s o(4,2))$. Here we consider the following Bruhat decomposition (consistent with the maximal cuspidal parabolic subalgebra):

$$
\mathcal{G}_{0}=\mathcal{G}_{1}^{+} \oplus \mathcal{M}_{1} \oplus \mathcal{A}_{1} \oplus \mathcal{G}_{1}^{-}
$$

where $\mathcal{M}_{1}=s o(2) \oplus s o(2,1), \mathcal{A}_{1}$ is one-dimensional, $\mathcal{G}_{1}^{+}, \mathcal{G}_{1}^{-}$are five-dimensional isomorphic subalgebras.

The signatures of the ERs in this case are [11]:

$$
\chi_{1}=\left\{n^{\prime}, k, \epsilon, \nu^{\prime}\right\}
$$

where $n^{\prime} \in \mathbb{Z}$ is a character of $s o(2), \nu^{\prime} \in \mathbb{C}$ is a character of $A_{1}, k, \epsilon$ fix a discrete series representation of $s o(2,1), k \in \mathbb{N}, \epsilon= \pm 1$, or a limit thereof when $k=0$.

The relation with the $\operatorname{sl}(4)$ Dynkin labels is as follows [11]:

$$
m_{1}=\frac{1}{2}\left(k-\nu^{\prime}+n^{\prime}\right), \quad m_{2}=-k, \quad m_{3}=\frac{1}{2}\left(k-\nu^{\prime}-n^{\prime}\right) .
$$

For the analysis we need the additional Harish-Chandra parameters:

$$
m_{12}=\frac{1}{2}\left(n^{\prime}-k-\nu^{\prime}\right), m_{23}=-\frac{1}{2}\left(k+\mu^{\prime}+n^{\prime}\right), m_{13}=-\nu^{\prime}
$$

We see that if $\nu^{\prime} \notin \mathbb{Z}$ then no Harish-Chandra parameter can be a positive integer, thus, the ERs would be irreducible. 
Thus, we consider the case $\nu^{\prime} \in \mathbb{Z}$. Actually, we shall use the analysis of the partially equivalent ERs in this case done in [11]. Thus, we use a parametrization taken from there (up to change of sign) by three positive integers: $p, \nu, n$, so that we have:

$$
\begin{aligned}
& \lambda_{p, \nu, n}=\left(m_{1}, m_{2}, m_{3}\right)_{p, \nu, n}=(-p-\nu, \nu,-n-\nu), \\
& m_{12}=-p, m_{23}=-n, m_{13}=-p-n-\nu
\end{aligned}
$$

It is known that when relating the root systems of $\mathcal{G}$ to $\mathcal{G}_{0}$ relative to the Bruhat decomposition (15) the root $\alpha_{2}$ is compact, the rest are non-compact, and the above parametrization is consistent with this.

\section{Parabolic Verma modules for $s l(4)$}

Here we enumerate the parabolic Verma modules for $s l(4)$. For this we need to produce the list of the various $\Delta_{ \pm}^{S}$ and the corresponding parabolic subalgebras. We have:

$$
\begin{aligned}
\Delta_{ \pm}^{\emptyset}= & 0, \quad \Delta_{ \pm}^{1}=\left\{ \pm \alpha_{1}\right\}, \quad \Delta_{ \pm}^{2}=\left\{ \pm \alpha_{2}\right\}, \quad \Delta_{ \pm}^{3}=\left\{ \pm \alpha_{3}\right\}, \quad \Delta_{ \pm}^{123}=\Delta_{ \pm}, \\
\Delta_{ \pm}^{12}= & \left\{ \pm \alpha_{1}, \pm \alpha_{2}, \pm \alpha_{12}\right\}, \quad \Delta_{ \pm}^{23}=\left\{ \pm \alpha_{2}, \pm \alpha_{3}, \pm \alpha_{23}\right\}, \quad \Delta_{ \pm}^{1,3}=\left\{ \pm \alpha_{1}, \pm \alpha_{3}\right\} \\
& P_{\emptyset}=\mathcal{B}, \quad P_{\{i\}}=\mathcal{G}_{-}^{i} \amalg \mathcal{B}, \quad(i=1,2,3) \\
& P_{\{12\}}=\mathcal{G}_{-}^{1} \amalg \mathcal{G}_{-}^{2} \amalg \mathcal{G}_{-}^{12} \amalg \mathcal{B}, \quad P_{\{23\}}=\mathcal{G}_{-}^{2} \amalg \mathcal{G}_{-}^{3} \amalg \mathcal{G}_{-}^{23} \amalg \mathcal{B}, \\
& P_{\{1,3\}}=\mathcal{G}_{-}^{1} \amalg \mathcal{G}_{-}^{3} \amalg \mathcal{B}, \quad P_{\{123\}}=\mathcal{G}_{-} \amalg \mathcal{B}=\mathcal{G}
\end{aligned}
$$

Now we can make connection with some generalized Verma modules.

In order to compare the parabolic subalgebras of the real form with the parabolic subalgebras of the complexification $\operatorname{sl}(4, \mathbb{C})$ we need the complexification of $(9)$. We have:

$$
\begin{aligned}
& \mathcal{P}_{0}^{\mathbb{C}}=\operatorname{so}(2, \mathbb{C}) \oplus \mathcal{A}_{0}^{\mathbb{C}} \oplus \mathcal{N}_{0}^{\mathbb{C}}, \\
& \mathcal{P}_{1}^{\mathbb{C}}=\operatorname{so}(2, \mathbb{C}) \oplus \operatorname{sl}(2, \mathbb{C}) \oplus \mathcal{A}_{1}^{\mathbb{C}} \oplus \mathcal{N}_{1}^{\mathbb{C}}, \\
& \mathcal{P}_{2}^{\mathbb{C}}=\operatorname{so}(4, \mathbb{C}) \oplus \mathcal{A}_{2}^{\mathbb{C}} \oplus \mathcal{N}_{2}^{\mathbb{C}}
\end{aligned}
$$

- First we note that $\mathcal{H} \cong s o(2, \mathbb{C}) \oplus \mathcal{A}_{0}^{\mathbb{C}}, \mathcal{N}_{0}^{\mathbb{C}} \cong \mathcal{N}$, thus: $\mathcal{P}_{0}^{\mathbb{C}}=\mathcal{B}$.

- Further, we record the triangular decomposition : $\operatorname{sl}(2, \mathbb{C})=\operatorname{sl}(2, \mathbb{C})^{+} \oplus \operatorname{sl}(2, \mathbb{C})_{h} \oplus \operatorname{sl}(2, \mathbb{C})^{-}$, where $\operatorname{sl}(2, \mathbb{C})_{h}$ is a Cartan subalgebra of $\operatorname{sl}(2, \mathbb{C})$. Then we note that $\mathcal{H} \cong s o(2, \mathbb{C}) \oplus$ $\operatorname{sl}(2, \mathbb{C})_{h} \oplus \mathcal{A}_{1}^{\mathbb{C}}, \mathcal{N} \cong \operatorname{sl}(2, \mathbb{C})^{+} \oplus \mathcal{N}_{1}^{\mathbb{C}}$, and thus we have:

$$
\mathcal{P}_{1}^{\mathbb{C}}=\operatorname{sl}(2, \mathbb{C})^{-} \oplus \mathcal{B} \cong P_{\{2\}}
$$

Now we can note that the GVM $V^{\Lambda\left(\chi_{1}\right)}$ with $\chi_{1}=\left\{n^{\prime}, k, \epsilon, \nu^{\prime}\right\}$ is isomorphic to PWM $V_{2}^{M\left(\lambda_{p, \nu, n}\right)}$, so that

$$
\lambda\left(h_{1}, h_{2}, h_{3}\right)=\left(m_{1}-1, m_{2}-1, m_{3}-1\right)=(-p-\nu-1, \nu-1,-n-\nu-1)
$$

- Next, we record the triangular decomposition : $s o(4, \mathbb{C})=s o(4, \mathbb{C})^{+} \oplus s o(4, \mathbb{C})_{h} \oplus s o(4, \mathbb{C})^{-}$, where $s o(4, \mathbb{C})_{h}$ is a Cartan subalgebra of $s o(4, \mathbb{C})$. Next we note that $\mathcal{H} \cong s o(4, \mathbb{C})_{h} \oplus \mathcal{A}_{2}^{\mathbb{C}}$. $\mathcal{N} \cong \operatorname{so}(4, \mathbb{C})^{+} \oplus \mathcal{N}_{2}^{\mathbb{C}}$, and thus we have:

$$
\mathcal{P}_{2}^{\mathbb{C}}=s o(4, \mathbb{C})^{-} \oplus \mathcal{B} \cong P_{\{1,3\}}
$$


Finally, we note that the GVM $V^{\Lambda(\chi)}$ with $\chi=\left[j_{1}, j_{2} ; d\right]$ is isomorphic to PWM $V_{1,3}^{M(\lambda)}$, where

$$
\lambda\left(h_{1}, h_{2}, h_{3}\right)=\left(m_{1}-1, m_{2}-1, m_{3}-1\right)=\left(2 j_{1}, 1-d-j_{1}-j_{2}, 2 j_{2}\right)
$$

\section{Minimal parabolics vs. complex parabolic subalgebras}

Here we briefly discuss the relation of minimal parabolics $\mathcal{P}_{0}=\mathcal{M}_{0} \oplus \mathcal{A}_{0} \oplus \mathcal{N}_{0}$ of classical real Lie algebras to the parabolic subalgebras of their complexification. (For the minimal parabolic subalgebras and the enumeration of simple roots we use info from [7,12].)

In the case of split real Lie algebras $\mathcal{G}_{r}$ we have the general fact that $\mathcal{M}_{0}=0$ and then the complexification of the minimal parabolic of $\mathcal{G}_{r}$ is isomorphic to the Borel subalgebra of $\mathcal{G}_{r}^{\mathbb{C}}$. (We recall also that in this case $\mathcal{A}_{0}^{\mathbb{C}} \cong \mathcal{H}\left(\mathcal{G}_{r}^{\mathbb{C}}\right)$.) For completeness we list the classical split real Lie algebras: $s l(n, \mathbb{R})$, so $(r, r)$, so $(r+1, r)$, $s p(n, \mathbb{R})$.

There are cases of non-split real Lie algebras $\mathcal{G}_{r}$ when the minimal parabolic is isomorphic to $\mathcal{B}\left(\mathcal{G}_{r}^{\mathbb{C}}\right)$. That is when the subalgebra $\mathcal{M}_{0}$ is abelian. Then $\mathcal{M}_{0}^{\mathbb{C}} \oplus \mathcal{A}_{0}^{\mathbb{C}} \cong \mathcal{H}\left(\mathcal{G}_{r}^{\mathbb{C}}\right)$. In the classical case this is the real Lie algebra $s u(n, n),(n>1)$, where we have: $\mathcal{M}_{0}=u(1) \oplus \cdots \oplus \cdots u(1)$, ( $n-1$ entries $), \operatorname{dim}_{\mathbb{R}} \mathcal{A}_{0}=n, \operatorname{dim}_{\mathbb{R}} \mathcal{N}_{0}=n(2 n-1)$. Then, $\mathcal{P}_{0}^{\mathbb{C}} \cong \mathcal{B}(\operatorname{sl}(2 n, \mathbb{C}))$.

Next we consider the rest of the real Lie algebras where the relation of the minimal parabolic to the complex parabolics is more involved.

In the case of $s u^{*}(2 n)(n>1)$ the minimal parabolic subalgebra is given by: $\mathcal{M}_{0}=s u(2) \oplus$ $\cdots \oplus \cdots s u(2),(n$ entries $), \operatorname{dim}_{\mathbb{R}} \mathcal{A}_{0}=n-1, \operatorname{dim}_{\mathbb{R}} \mathcal{N}_{0}=2 n(n-1)$. Thus, $\mathcal{P}_{0}^{\mathbb{C}} \cong P_{1,3, \ldots, 2 n-1} \cong$ $\amalg_{i=1}^{n} \mathcal{G}_{-}^{2 i-1} \amalg \mathcal{B}$.

In the case of $s u(p, r)(p>r \geq 1)$ the minimal parabolic subalgebra is given by: $\mathcal{M}_{0}=s u(p-r) \oplus u(1) \oplus \cdots \oplus \cdots u(1),(r$ entries $), \operatorname{dim}_{\mathbb{R}} \mathcal{A}_{0}=r, \operatorname{dim}_{\mathbb{R}} \mathcal{N}_{0}=r(2 p-1)$. Thus, $\mathcal{P}_{0}^{\mathbb{C}} \cong P_{r+1, \ldots, p-1} \cong \amalg_{i=r+1}^{p-1} \mathcal{G}_{-}^{i} \amalg \mathcal{B}$.

In the case of $s o(p, r)(p>r+1)$ the minimal parabolic subalgebra is given by: $\mathcal{M}_{0}=s o(p-$ $r), \operatorname{dim}_{\mathbb{R}} \mathcal{A}_{0}=r, \operatorname{dim}_{\mathbb{R}} \mathcal{N}_{0}=r(p-1)$. Thus, $\mathcal{P}_{0}^{\mathbb{C}} \cong P_{r+1, \ldots,[(p+r) / 2]} \cong \amalg_{i=r+1}^{[(p+r) / 2]} \mathcal{G}_{-}^{i} \amalg \mathcal{B}$.

Next we consider $s p(p, r) \quad(p \geq r)$. The minimal parabolic subalgebra is given by: $\mathcal{M}_{0}=s p(p-r) \oplus s p(1) \oplus \cdots \oplus s p(1),(r$ factors $), \operatorname{dim}_{\mathbb{R}} \mathcal{A}_{0}=r, \operatorname{dim}_{\mathbb{R}} \mathcal{N}_{0}=r(4 p-1)$. In the case $p=r$ we have $\mathcal{P}_{0}^{\mathbb{C}} \cong P_{1,3, \ldots, 2 r-1} \cong \amalg_{i=1}^{r} \mathcal{G}_{-}^{2 i-1} \amalg \mathcal{B}$. In the case $p>r$ we have $\mathcal{P}_{0}^{\mathbb{C}} \cong P_{1,3, \ldots, 2 r-1,2 r+1,2 r+2, \ldots, p+r} \cong \amalg_{i=1}^{r} \mathcal{G}_{-}^{2 i-1} \amalg_{j=2 r+1}^{p+r} \mathcal{G}_{-}^{j} \amalg \mathcal{B}$.

Finally, we consider $s o^{*}(2 n)$. First we suppose $n=2 r$. Then $\mathcal{M}_{0}=s o(3) \oplus \cdots \oplus s o(3),(r$ factors $), \operatorname{dim}_{\mathbb{R}} \mathcal{A}_{0}=r, \operatorname{dim}_{\mathbb{R}} \mathcal{N}_{0}=r(4 r-3)$. Note also that $\mathcal{M}_{0 h}^{\mathbb{C}} \oplus \mathcal{A}_{0}^{\mathbb{C}} \cong \mathcal{H}(\operatorname{so}(2 n, \mathbb{C})$.

Thus, we have $\mathcal{P}_{0}^{\mathbb{C}} \cong P_{1,3, \ldots, n-1} \cong \amalg_{i=1}^{r} \mathcal{G}_{-}^{2 i-1} \amalg \mathcal{B}$.

Next we suppose $n=2 r+1$. Then $\mathcal{M}_{0}=s o(2) \oplus s o(3) \oplus \cdots \oplus s o(3),(r$ factors $), \operatorname{dim}_{\mathbb{R}} \mathcal{A}_{0}=r$, $\operatorname{dim}_{\mathbb{R}} \mathcal{N}_{0}=r(4 r+1)$. Thus, we have $\mathcal{P}_{0}^{\mathbb{C}} \cong P_{1,3, \ldots, n-2} \cong \amalg_{i=1}^{r} \mathcal{G}_{-}^{2 i-1} \amalg \mathcal{B}$

For the lack of space we leave consideration of the exceptional real Lie algebras for a subsequent publication [13].

Discussion: Another main ingredient of our approach as follows. We group the (reducible) ERs with the same Casimirs in sets called multiplets [3]. The multiplet corresponding to fixed values of the Casimirs may be depicted as a connected graph, the vertices of which correspond to the reducible ERs and the lines (arrows) between the vertices correspond to intertwining operators. The explicit parametrization of the multiplets and of their ERs is important for understanding of 
the situation. The notion of multiplets was introduced in [14]. Then it was applied to (infinitedimensional) (super-)algebras, quantum groups and other symmetry objects. For a current summary of these developments, see [7,15], for further developments - [13].

Acknowledgment. The author has received partial support from Bulgarian NSF Grant DN-18/1.

\section{References}

[1] Dobrev V. K., Mack G., Petkova V. B., Petrova S. G., Todorov I. T. Harmonic Analysis on the $n$-Dimensional Lorentz Group and Its Applications to Conformal Quantum Field Theory. Lecture Notes in Physics. V. 63. Berlin: Springer 1977.

[2] Knapp A.W. Representation Theory of Semisimple Groups (An Overview Based on Examples.) Princeton Univ. Press 1986.

[3] Dobrev V. K. Canonical construction of intertwining differential operators associated with representations of real semisimple Lie groups // Rept. Math. Phys. 1988. V. 25 P. 159-181.

[4] Dixmier J. Enveloping Algebras. New York: North Holland 1977.

[5] Verma N. Structure of certain induced representations of complex semisimple Lie algebras. // Bull. Amer. Math. Soc. 1968. V. 74.

[6] Lepowsky J. Generalized Verma modules, the Cartan-Helgason theorem, and the HarishChandra Homomorphism // J. Algebra 1977. V. 49. P. 470-495.

[7] Dobrev V. K. Invariant Differential Operators, Volume 1: Noncompact Semisimple Lie Algebras and Groups. De Gruyter Studies in Mathematical Physics. V. 35. Berlin: De Gruyter. 2016.

[8] Bernstein I. N., Gel'fand I. M., Gel'fand S. I. Structure of representations generated by highest weight vectors // Funkts. Anal. Prilozh. 1971. V. 5. P. 1-9.

[9] Bruhat F. Sur les representations induites des groups de Lie // Bull. Soc. Math. France. 1956. V. 84. P. 97-205.

[10] Harish-Chandra Discrete series for semisimple Lie groups: II // Ann. Math. 1966. V. 116. P. 1-111.

[11] Dobrev V. K. Classification of Conformal Representations Induced from the Maximal Cuspidal Parabolic // Physics of Atomic Nuclei. 2017. V. 80. P. 347352.

[12] Dobrev V. K. Invariant Differential Operators for Non-Compact Lie Groups: Parabolic Subalgebras // Rev. Math. Phys. 2008. V. 20. P. 407-449.

[13] Dobrev V. K. In preparation.

[14] Dobrev V. K. Multiplet classification of the reducible elementary representations of real semi-simple Lie groups: the $S O_{e}(p, q)$ example // Lett. Math. Phys. 1985. V. 9. P. 205-211; Elementary representations and intertwining operators for $S U(2,2)$ : I // J. Math. Phys. 1985. V. 26. P. 235-251.

[15] Dobrev V. K. Invariant Differential Operators, Volume 2: Quantum Groups. Volume 3: Supersymmetry. Volume 4: AdS/CFT, (Super-)Virasoro and Affine (Super-)Algebras. De Gruyter Studies in Mathematical Physics V. 39. V. 49. V. 53. Berlin: De Gruyter. 2017. 2018. 2019. 